\title{
Influence of Polarization on Keyhole Probability on a MIMO-OFDM Train-to-Wayside System on Tunnels
}

\author{
Juan Moreno García-Loygorri, \\ Leandro De Haro, \\ Luis Cuéllar, and José Manuel Riera,
}

Carlos Rodríguez,

\begin{abstract}
This letter presents a deep insight on a real implementation of a train-to-wayside broadband radio on subway tunnels that makes use of a $2 \times 2$ MIMO-OFDM setup. A keyhole is a phenomenon that usually happens in tunnels and that seriously degrades channel's capacity, even if both transmitter and receiver antennas are uncorrelated. The main purpose of this letter is to study in detail the influence of the polarization in the probability of having a keyhole on a MIMO-OFDM train-to-wayside communication system on a tunnel. MIMO keyholes are studied in four different polarization setups, six different tunnels cross-sections and, finally, capacity results are provided.
\end{abstract}

\section{INTRODUCTION}

A S THE competition between different types of transports gets fiercer, the focus on the technologies that can provide a mark-up increases. Among these technologies are those related to security (on-board CCTV), safety (railway signaling systems, like CBTC), or commercial applications (Internet access for travelers, customer information, etc.). Most of them have strong needs in terms of bandwidth, delay, or jitter, and the existing technologies in transport systems, like TETRA and GSM-R, cannot address them [1]

The solution may be the use of one of the technologies that fulfill the 4th generation mobile communication IMT-A requirements specified by the ITU-R, like LTE or WiMAX [2]. Among them, LTE seems the most likely to be the next standard for mobile railway communications [3]. MIMO is one of the fundamental technologies that make possible the high data rates that LTE provides, even in hard environments like tunnels [4].

So, MIMO seems to be a fine solution for broadband radios, mostly in hard environments, but it is not entirely unproblematic.

J. Moreno and C. Rodríguez are with the Engineering Department, Metro de Madrid S.A., 28007 Madrid, Spain (e-mail: juan.moreno@metromadrid.es; carlos@metromadrid.es).

L. de Haro, L. Cuéllar, and J. M. Riera are with the Universidad Politéenica de Madrid (UPM), ETSI Telecomunicación, Ciudad Universitaria, 28040 Madrid, Spain (e-mail: leandro.deharo.ariet@upm; esluiscn@gr.ssr.upm.es; jm.riera@upm.es).
In tunnels and other scenarios like roof edges, it is possible to have low spatial correlation and a low rank in the channel matrix [5]. This is the academic definition of keyhole, and the tunnel scenario was identified from the very beginning [5] as one of the most likely to have keyholes. This physical phenomenon leads to a substantial decrease in the channel capacity, so it is really necessary to study it before deploying any MIMO system on a tunnel environment

Because the influence of polarization diversity on correlation and capacity is well known [6], in this letter we focus on the influence of the polarization diversity on keyhole probability.

It is almost impossible to properly address every contribution related to MIMO in tunnels. But we need to reference at least the most important work of this field: a pioneer paper, but on narrowband measurements [7]; another one focused on polarization effects in tunnels [8]; broadband measurements [9], [10] but all of them with no train involved, this is with both transmitter and receiver placed in the ground. For the purpose of this letter, we also need to mention the classical paper on keyholes that provides the theoretical background [5] and a more recent one, focused on measurements of keyholes [11]. Moreover, this letter clearly identifies tunnels as a suitable scenario to have keyholes. Finally, in a previous letter [12], we presented keyhole probabilities and their influence on capacity on many radically different tunnels built up with different constructive methods. Here we go deep into our previous work in order to explain the influence of polarization on keyholes, considering some different tunnel crosssections. The most important fact is that, as far as we know, there are no broadband MIMO measurements in tunnels

This letter is organized as follows. In Section II the measurements' setup and the keyhole and channel estimation procedure are depicted; in Section III we present the obtained results and, finally, in Section IV conclusions are given.

\section{SetuP AND PARAmeter Estimation}

\section{A. Setup. Testbed and Antenna Array.}

To perform these measurements, a testbed already developed by some members of this group was employed [13]. The testbed implements both a DVB-T2 transmitter [14] and a receiver. This was motivated because the transmission technology for DVB-T2 is OFDM, the same as in LTE's downlink. This testbed implements the frame structure of DVB-T2, but slightly modified from $2 \times 1$ MISO to a $2 \times 2$ MIMO. Table I shows the principal parameters of the setup.

Regarding the antennas, in this research work we had arrays composed by short dipoles (each antenna was $22 \mathrm{~cm}$ long), and every antenna was well matched at the desired frequency band 


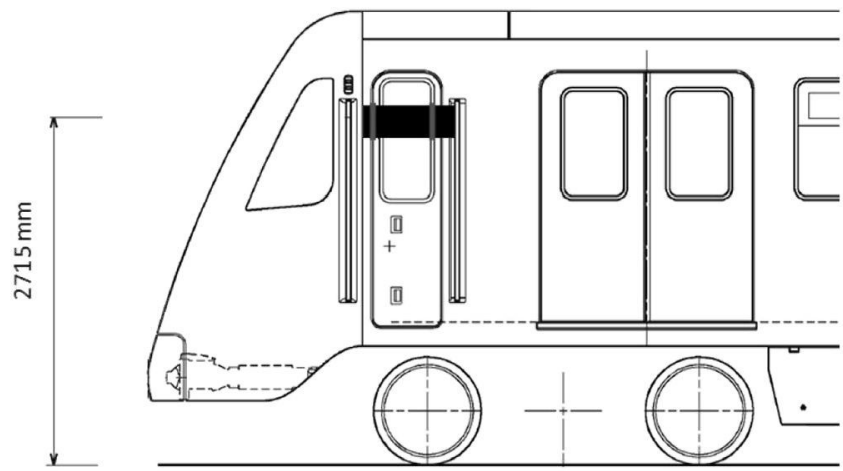

Fig. 1. The antenna array was located in the cabin's window over a dielectric surface (depicted in black).

TABLE I

Main Parameters of THE MiMO Testbed

\begin{tabular}{ccc}
\hline \hline Parameter & Symbol & Value \\
\hline FFT mode & - & $2 \mathrm{~K}$ \\
Guard Interval & $\mathrm{GI}$ & $1 / 8$ \\
Scattered pilot pattern & & $\mathrm{PP} 1$ \\
Modulation & - & $64 \mathrm{QAM}$ \\
Sampling frequency & $\mathrm{F}_{\mathrm{s}}$ & $9.1429 \mathrm{MHz}$ \\
Useful symbol time & $\mathrm{T}_{\mathrm{u}}$ & $2048 / \mathrm{F}_{\mathrm{s}}=224 \mu \mathrm{s}$ \\
Guard time & $\mathrm{T}_{\mathrm{g}}$ & $\mathrm{T}_{\mathrm{s}} / 8=28 \mu \mathrm{s}$ \\
Symbol time & $\mathrm{T}_{\mathrm{s}}$ & $252 \mu \mathrm{s}$ \\
Bandwidth & $\mathrm{BW}$ & $8 \mathrm{MHz}$ \\
Data subcarriers & $\mathrm{N}_{\mathrm{d}}$ & 1878 \\
Carrier spacing & $\Delta \mathrm{f}$ & $4.26 \mathrm{KHz}$ \\
\hline \hline
\end{tabular}

(594 MHz). This frequency was chosen because it is close to both lower LTE bands and to other railway-related bands. We placed the on-board array in the cabin's window (see Fig. 1), because in real scenarios it is almost impossible to locate the antennas in the best places from the propagation point of view (probably, the windshield). Way side antennas were placed at the end of the platform, at a height of $0.95 \mathrm{~m}$, and the axis of the array formed an angle of $30^{\circ}$ with the axis of the tunnel. Spacing of the elements within the antenna array was $\lambda(51 \mathrm{~cm})$ on both arrays. In [15], some results related to antenna placement for train-to-wayside communications can be found.

\section{B. Data Model and Channel Estimation}

To estimate the channel matrix $\mathbf{H}$, we followed the same steps as in our previous papers [12][13]. The data model is shown in (1), where $\mathbf{X}_{\mathbf{k}}$ represents the transmitted data symbol vector (for the $\boldsymbol{k}$ th subcarrier), $\mathbf{N}_{\mathbf{k}}$ is the noise vector related to subcarrier $\boldsymbol{k}$, $\mathbf{H}_{\mathbf{k}}$ is the MIMO channel matrix, and $\mathbf{Y}_{\mathbf{k}}$ is the received vector. So, at any time $t$ we have $k \mathbf{H}_{\mathrm{k}}$ matrices to estimate.

$$
Y_{k}=H_{k} X_{k}+N_{k}
$$

To normalize $\mathbf{H}_{\mathbf{k}}$ we considered the average power of the OFDM symbol $\mathbf{H}\left(t_{l}\right)$ on every subcarrier of the symbol (2). So, for the $l$ th symbol for every subcarrier $k$, we have:

$$
h_{i j_{k, l}}=\frac{h_{i j}\left(t_{l}, f_{k)}\right.}{\left\|H\left(t_{l}, f\right)\right\|}
$$

where $i j$ are the $2 \times 2$ elements of the $\mathbf{H}_{\mathbf{k}}$ matrix.
If we suppose equal power allocation for each subchannel, capacity can be computed by the classical equation [4] for MIMO channels (3):

$$
C_{U P}=\log _{2}\left[\operatorname{det}\left(I_{M}+\frac{S N R}{N} H H^{H}\right)\right]
$$

where $M$ and $N$ are the number of receiving and transmitting antenna elements, respectively, and $\mathbf{H}^{\mathbf{H}}$ means the transpose conjugate of $\mathbf{H}$.

\section{Keyhole Estimation}

On a diversity scenario, the higher the correlation, the lower the channel capacity. The academic definition for a keyhole is based on the rank of the channel matrix. If we have a low spatial correlation between antennas and, unexpectedly, channel matrix $\mathbf{H}$ has a low rank (ideally equal to 1), we have a keyhole [5]. The best example of this is a wall (with a narrow hole) between the transmitting array and the receiving array.

To properly estimate the probability of having a keyhole, we need to decouple the influence of the correlation between elements in the array and the keyholes. The highest measured correlation between the elements of the array for all the scenarios described in this letter is 0.712 , very close to 0.7 , which means that it does not play an important role [16]. If the rank of the channel matrix is close to 1 , we will only ascribe this to the presence of a keyhole.

Due to the fact that the presence of noise in the transmission (and hence, in the estimation of $\mathbf{H}$ ) makes impossible to estimate the presence of a keyhole computing the rank of the matrix, we need to find a more practical approach to compute keyholes. This procedure comes from [11] and it is summarized in (4):

$$
\frac{\max \left(\lambda_{1}, \lambda_{2}\right)}{\min \left(\lambda_{1}, \lambda_{2}\right)}=\left\{\begin{array}{ll}
>S N R & \rightarrow \text { keyhole } \\
\leq S N R & \rightarrow \text { no keyhole }
\end{array}\right\}
$$

where $\lambda_{1}$ and $\lambda_{2}$ are the two eigenvalues of the $2 \times 2$ channel matrix $\mathbf{H}$.

\section{EXPERIMENTAL RESUlts}

\section{A. Measurements}

Using the MIMO testbed described in Section II, we carried out a measurement campaign on the subway network in Madrid, Spain. In Fig. 2 the cross-section of the tunnels is shown. We only include the old-tunnel part of the previous letter, because we measured almost no keyhole in the new-tunnel part. So this part is consequently omitted here.

We made sure that every measurement was carried out under NLOS conditions, and the train ran from one station to the next one where the wayside antenna was placed.

Every measurement was performed at real in-operation conditions, all of them at the same speed $( \pm 2 \mathrm{~km} / \mathrm{h})$, because the speed of the rolling stock is accurately controlled by the on-board signaling system. To properly estimate the influence of polarization on keyhole probability, we took measurements in four different scenarios (see Table II). Each probability provided is the average of four sets of independent measurements. The measured SNR was $20 \mathrm{~dB}$. 


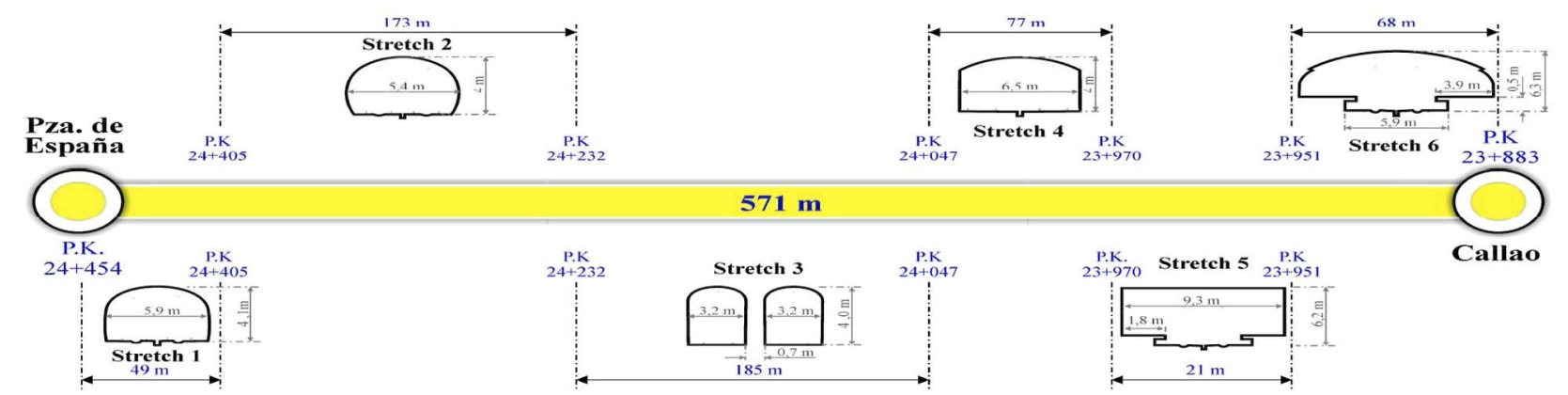

Fig. 2. Old tunnel cross-sections (Callao-Plaza de España).

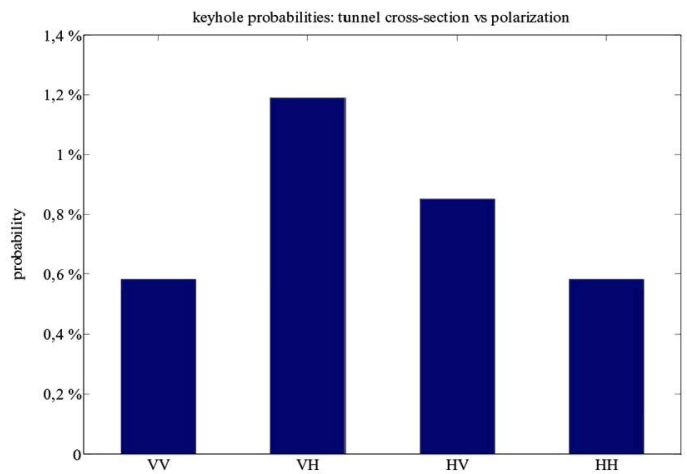

Fig. 3. Average absolute keyhole probability for VV, VH, HV, and HH polarizations. Antenna spacing between array elements is $\lambda$.

TABLE II

POLARIZATIONS FOR EACH SCENARIO

\begin{tabular}{cccc}
\hline \hline ID & Transmitter & Receiver & Abbreviation \\
\hline 1 & Vertical & Vertical & VV \\
2 & Horizontal & Vertical & HV \\
3 & Vertical & Horizontal & VH \\
4 & Horizontal & Horizontal & HH \\
\hline \hline
\end{tabular}

\section{B. Results}

As we stated before, in this letter we provide two different results: MIMO capacity and keyhole statistics related to polarization and tunnel cross-section. In Fig. 3 we can see the absolute probabilities of having a keyhole on each one of the four considered polarizations (VV, VH, HV, and $\mathrm{HH}$ ). In Fig. 4, we provide the same result but disaggregated on each tunnel cross-section.

The average keyhole probability of all these stretches was characterized in our previous paper [12], where we found that the sections more likely to have keyholes were stretches 2 and 3 (see [12, Fig. 2]).

In order to extract proper conclusions of the performance of the four different polarization scenarios, a suitable parameter could be the capacity exceeded in $90 \%$ of the measurements $\left(\mathbf{C}_{\mathbf{9 0}}\right)$, which is shown in Table III for all the stretches and polarization combinations. Looking at the values of Table III, we can see that we have better $\mathbf{C}_{90}$ capacities in $\mathrm{HV}$ and VV than $\mathrm{VH}$ and $\mathrm{VH}$, due to the fact that horizontally polarized modes suffer higher attenuation in curved tunnels [16]. This result agrees with the measured results on polarization and

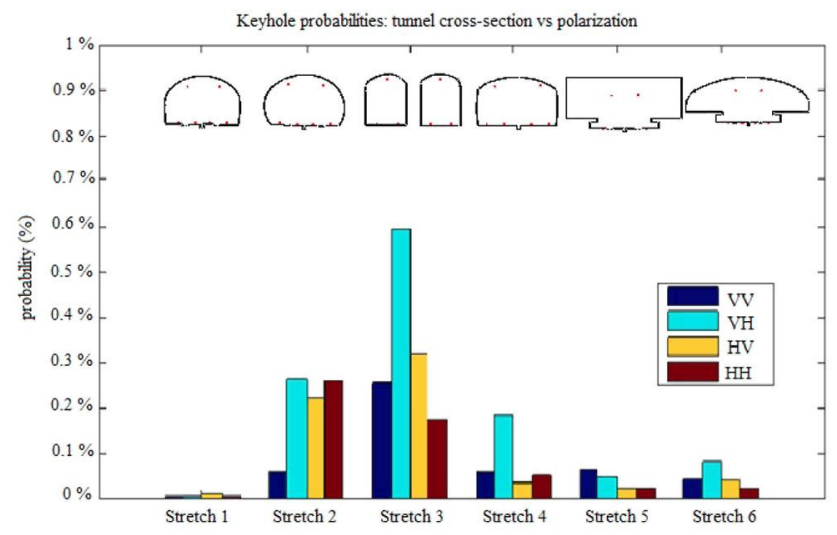

Fig. 4. Average absolute keyhole probability for each tunnel cross-section (VV, $\mathrm{VH}, \mathrm{HV}$, and HH polarizations). These data sets are provided in Table IV.

TABLE III

$\mathbf{C}_{\boldsymbol{9} 0}$ Values for VV, VH, HV, and HH Polarizations (IN BPs/Hz). THese Results are Aggregated for Ald Tunnel Cross-Sections in Fig. 2

\begin{tabular}{crc}
\hline \hline Transmitter/Receiver & Vertical & Horizontal \\
\hline Vertical & 7.23 & 6.82 \\
Horizontal & 7.15 & 6.78 \\
\hline \hline
\end{tabular}

cross-polarization of other research work [17] but this one does not consider keyholes.

Starting with all the stretches together, these probabilities provide some interesting results. The most important one is that the VH case is the most likely to have keyholes, distantly followed by HV, and finally, by both $\mathrm{HH}$ and VV.

Another significant result is that $\mathrm{HH}$ and VV are (on average) almost equally likely to have keyholes, and this value is close to half that of the VH case. All of these results lead to the fact that polarization diversity in a tunnel does not always imply a capacity improvement due to keyholes, at least partly

In the worst case $(\mathrm{VH})$, the average keyhole probability is near $1.2 \%$, almost twice the non-diversity polarizations (VV and $\mathrm{HH}$, with a probability of $0.58 \%$ ). This is mostly due to the propagation characteristics of stretch 3 (a small arched tunnel, far from the rectangular one, where this $\mathrm{VH}$ scenario would achieve better results) which concentrates the majority of the keyholes (see Fig. 4 and Table IV). The HV case has a $0.85 \%$ probability of having a keyhole.

In Fig. 4, we show the average keyhole probabilities for each measured tunnel cross-section. If we consider the detailed results for each stretch, HH keyhole probabilities remain close to 
TABLE IV

Average Keyhole Probabilities for Each TunNel Stretch and POLARIZATION

\begin{tabular}{ccccc}
\hline \hline Tunnel stretch & VV & VH & HV & HH \\
\hline 1 & $0.06 \%$ & $0.05 \%$ & $0.12 \%$ & $0.07 \%$ \\
2 & $0.57 \%$ & $2.65 \%$ & $2.23 \%$ & $2.59 \%$ \\
3 & $2.56 \%$ & $5.94 \%$ & $3.22 \%$ & $1.73 \%$ \\
4 & $0.57 \%$ & $1.83 \%$ & $0.34 \%$ & $0.53 \%$ \\
5 & $0.63 \%$ & $0.48 \%$ & $0.24 \%$ & $0.26 \%$ \\
6 & $0.41 \%$ & $0.81 \%$ & $0.40 \%$ & $0.22 \%$ \\
\hline \hline
\end{tabular}

the VV case in almost every scenario. We see that the VH case is especially prone to keyholes and stretch 3 (the two-tunnel one) concentrates a significant amount of keyholes.

These results are coherent with the keyhole probabilities previously discussed, since the larger capacities are associated with smaller probabilities of having a keyhole. However, the observed difference in terms of keyhole probability between $\mathrm{VH}$ and HV does not lead to a significant difference of channel capacity between these two cases. This is because the worst keyhole probability is less than $6 \%$, so the impact on the average capacity is not very large.

Finally, in Table IV we put together the keyhole probabilities for every polarization and tunnel cross-section.

Stretch 3 has the highest keyhole probability in almost every polarization scenario, and the $\mathrm{VH}$ case gets the highest one on this tunnel stretch (with a significant 5.9\%) and also in the others.

As we stated before, VH (and HV) scenarios achieve worse results on arched or even circular tunnels, but in rectangular tunnels, VH or HV (depending on the aspect ratio of the rectangle) will obtain better results in terms of capacity. This is shown in Table IV, tunnel V (almost rectangular), where VH and HV achieve better results than the arched tunnels.

\section{CONClusion}

The performance of a MIMO-OFDM system has been evaluated with a measurement campaign carried out in the subway of Madrid, Spain. These measurements were carried out at $594 \mathrm{MHz}$, using a train and in real in-operation conditions.

Keyhole probabilities have been related to four different polarization configurations and the results were coherent with the average channel capacities. Moreover, we mapped the influence of the tunnel cross-section with the keyhole probability for every polarization scenario. The VH case has emerged as the worst case in terms of keyhole probability $(1.2 \%$ in average and close to $6 \%$ in one of the stretches). This has been ascribed to the strong influence of tunnel stretches 3 and 4 , because it is here where this VH scenario detaches from the others in terms of keyhole probability. We identify two key parameters that influence this probability: shape of the tunnel and changes in the cross-section itself, and stretches 2, 3, and 4 (especially stretch 3) have more changes in cross-section than the others. Given that the measured keyhole probabilities are very short, their influence on capacity is only relevant in some realizations, but not in the whole intersection. That is, the impact of the keyhole phenomenon in tunnels is limited, and it is mitigated in part by the OFDM modulation.

\section{REFERENCES}

[1] A. Bertout, "Changing track: Ground-to-train broadband communications and new train-borne applications are considering a move to 4G/LTE," Railway Strategies Oct--Nov. 2009 [Online]. Available: http://www.railwaystrategies.co.uk

[2] International Telecommunication Union (ITU), "Requirements related to technical performance for IMT-Advanced radio interface(s)," Report ITU-R M.2134., 2008 [Online]. Available: http://www.itu.int/ $\mathrm{dms} \_$pub/itu-r/opb/rep/R-REP-M.2134-2008-PDF-E.pdf

[3] UIC, "LTE/SAE-The future railway mobile radio system? Long-term visions on railway mobile radio technologies," V 0.4 Draft 14.09.2009, 2009.

[4] A. Goldsmith, S. Ali Jafar, N. Jindal, and S. Vishwanath, "Capacity limits of MIMO Channels," IEEE J. Sel. Areas Commun., vol. 21, no. 5, pp. 684-702, Jun. 2003.

[5] D. Chizik, G. Foschini, M. Grans, and R. Valenzuela, "Keyholes, correlations and capacities of multielement transmit and receive antennas," IEEE Trans. Wireless Commun, vol. 1, no. 2, pp. 361-368, Apr. 2002.

[6] J. F. Valenzuela-Valdes et al., "The role of polarization diversity for MIMO systems under Rayleigh-fading environments," IEEE Antennas Wireless Propag. Lett., vol. 5, pp. 534-536, 2006.

[7] M. Lienard, P. Degauque, J. Baudet, and D. Degardin, "Investigation on MIMO channels in subway tunnels," IEEE J. Sel. Areas Commun., vol. 21, no. 3, pp. 332-339, Apr. 2003.

[8] J. M. Molina-Garcia Pardo, M. Lienard, P. Degauque, C. G. Pardo, and L. J. Llacer, "MIMO channel capacity with polarization diversity in arched tunnels," IEEE Antennas Wireless Propag. Lett., vol. 8, pp. $1186-1189,2009$.

[9] C. Sanchís-Borrás, J. M. Molina-García Pardo, M. Lienard, and P. Degauque, "Performance evaluation of MIMO-OFDM in tunnels," IEEE Antennas Wireless Propag. Lett., vol. 11, pp. 301-304, 2012.

[10] E. Masson et al., " $4 \times 4$ MIMO channel sounding in tunnels for trainto-wayside communications," in Proc. Int. Conf. Wireless Commun. Umusual Confined Areas, Aug. 28-30, 2012, pp. 1-5.

[11] P. Almers, F. Tuvfesson, and A. F. Molisch, "Keyhole effect in MIMO wireless channels: Measurements and theory," IEEE Trans. Wireless Commun., vol. 5, no. 12, pp. 3596-3604, Dec. 2006.

[12] J. Moreno, L. de Haro, C. Rodríguez, L. Cuéllar, and J. M. Riera, "Keyhole estimation of a MIMO-OFDM train-to-wayside communication system on subway tunnels," IEEE Antennas Wireless P ropag. Lett., vol. 14, pp. 88-91, 2015.

[13] C. Gómez-Calero, L. Cuéllar, L. de Haro, and R. Martínez, "A $2 \times 2$ MIMO DVB-T2 system: Design, new channel estimation scheme and measurements with polarization diversity," IEEE Trans. Broadcast., vol. 56 , no. 2 , pp. 184-192, Jun. 2010.

[14] ETSI, "Digital Video Broadcasting (DVB); Frame structure channel coding and modulation for a second generation digital terrestrial television broadcasting system (DVB-T2) EN 302755 V1.1.1, Apr. 2008.

[15] J. A. Castiblanco, D. Seetharamdoo, M. Berbineau, M. Ney, and F. Gallee, "Determination of antenna specification and positioning for efficient railway communication in tunnels of arbitrary cross section," in Proc. 11th ITST, 2011, pp. 678-683.

[16] D. Shiu, J. G. Foschini, and J. G. Gans, "Fading correlation and its effects on the capacity of multielement antenna systems," IEEE Trans. Commun., vol. 48, no. 3, pp. 502-513, Mar. 2000.

[17] J. A. Valdesueiro, B. Izquierdo, and J. Romeu, "On $2 \times 2$ MIMO observable capacity in subway tunnels at C-band: An experimental approach," IEEE Antennas Wireless Propag. Lett., vol. 9, pp. 1099-1102, 2010 